\title{
Current knowledge on the background, pathophysiology, and treatment of levodopa-induced dyskinesia - literature review.
}

\author{
Michał Hutny 1,", Jagoda Hofman ${ }^{1}$, Aleksandra Klimkowicz-Mrowiec ${ }^{2}$ and Agnieszka Gorzkowska ${ }^{3}$ \\ 1 Students' Scientific Society, Department of Neurorehabilitation, Faculty of Medical Sciences in Katowice, \\ Medical University of Silesia, 40-752 Katowice, Poland; michal.j.hutny@gmail.com \\ 2 Department of Internal Medicine and Gerontology, Faculty of Medicine, \\ Jagiellonian University, Medical College, Kraków, Poland; Aleksandra.Klimkowicz@mp.pl \\ 3 Department of Neurorehabilitation, Medical University of Silesia, \\ Faculty of Medical Sciences, School of Medicine, Katowice, Poland; agorzkowska@sum.edu.pl \\ * Correspondence: michal.j.hutny@gmail.com
}

\begin{abstract}
Levodopa remains the primary drug for controlling motor symptoms in Parkinson's disease through the whole course, but over time complications develop in the form of dyskinesias, which gradually become more frequent and severe. These abnormal, involuntary, hyperkinetic movements are mostly characteristic of the ON phase and reflect an excess of exogenous levodopa. They may also occur during OFF phase, or in both phases. Over the past 10 years, the issue of levodopa-induced dyskinesia has been the subject of research into both the substrate of this pathology and potential remedial strategies. The purpose of the present study was to review the results of recent research on the background and treatment of dyskinesia. To this end, databases were reviewed using a search strategy that included both relevant keywords related to the topic and appropriate filters to limit results to English-language literature published since 2010. Based on the selected papers, the current state of knowledge on morphological, functional, genetic, and clinical features of levodopa-induced dyskinesia, as well as pharmacological, genetic treatment and other therapies such as deep brain stimulation are described.
\end{abstract}

Keywords: Parkinson's disease; abnormal involuntary movements; dopaminergic signaling; basal ganglia; spiny projection neurons; neurotransmission; deep brain stimulation

\section{Introduction}

Treatment of Parkinson's disease (PD) is inseparable from the use of levodopa, 1-3,4dihydroxyphenylalanine (L-DOPA). Physiologically it is a precursor molecule in the synthesis of catecholamines that act as neurotransmitters in nervous system - epinephrine, norepinephrine (NE) and dopamine (DA). Decreased levels of DA, associated with a degeneration process in the substantia nigra (SN), lead to development of PD [1]. The first symptoms of PD appear when DA levels in the striatum are reduced by $80 \%$, with a corresponding neuronal DA loss of 60\% [2]. During this period, treatment is in "the honeymoon phase". L-DOPA administration alleviates motor symptoms of PD, such as bradykinesia and rigidity, without the presence of abnormal involuntary movements (AIMs). As the disease progresses, DA biosynthesis and storage decreases. When the dose of LDOPA entering the brain across the blood-brain barrier exceeds dopamine storage capacity of dopaminergic neurons, there is an increased dopaminergic activity, leading to hyperkinetic movements - levodopa-induced dyskinesias (LIDs), which typically appear about 5-6 years after PD diagnosis [1,3]. They occur at peak dose, with an increase or decrease in drug concentration, or both, which is referred to as biphasic LID. The DA level required to induce LID is lower with the duration of therapy [4].

Motor complications in PD patients develop after varying lengths of therapy. It is estimated that after initiation of levodopa treatment, $\sim 10 \%$ of patients per year develop movement fluctuations (Marsden and Parkes, 1977) and over 50\% of patients develop LID 
after 5 years of L-DOPA treatment [3]. This fact naturally raises the question - what are the determinants of their occurrence and severity? Another important question is whether there are any methods by which PD patients can be helped to improve their quality of live. This article attempts to answer these questions by reviewing the literature published on this topic in the last decade

\section{.2. Materials and Methods}

Database searches (PubMed, PubMed Central, Medline) were conducted over a 4 month period, from November 2020 to February 2021. The search strategy was based on creating input keywords consisting of "levodopa-induced dyskinesia" and related terms: "genetic", "therapeutic", "background", "pathogenesis" "therapy", "treatment", "deep brain stimulation", "dopamine receptor", "dopaminergic activity" and "signal". The filters applied to the results limited them to English-language papers published since 2010. Reviews, systematic reviews, personal communication, letters to the editor, conference material, and case reports were not included in the study. The online Mendelian Inheritance in Men database was used as a reference for gene loci and terminology.

\section{Results}

The results of the research were divided into sections concerning background (structural and functional; genetic; clinical) and treatment (pharmacological; genetic; invasive and noninvasive brain stimulation).

\subsection{Structural and functional background}

\subsubsection{Grey and white matter}

From the very beginning of the PD, magnetic resonance imaging (MRI) shows anatomical changes in the brain. These involve white matter hyperintensities (WMHs), and grey matter changes in the size of striatum. Both of these phenomena were examined for the significance of their intensity to the development of LID.

In animal model studies comparing severely dyskinetic 6-OHDA-lesioned rats with saline treated rats, it was shown that striatum size as well as asymmetry between sides increased with increasing duration of L-DOPA treatment, with a predominance on the lesioned side. The degree of asymmetry between sites in the size of the striatum and caudate nucleus at the onset of PD was found to be a predictor of the development of LID, but also of response to L-DOPA treatment [3]. The microscopic changes, although evident, did not appear to be directly related to striatal growth or the axial, limb and orolingual AIMs scores, measured together using established criteria of amplitude and severity [5].

Primary motor cortex (M1) in animals has also been shown to undergo changes associated with PD progression. In rats that developed LID, an increase in the density of dendritic spines was observed that was significantly correlated with the severity of LID. This fact indicates hypersensitivity to glutamatergic input of the neurons tested. On the contrary, the density of dendritic spines was not related to LID and increased with the duration of L-DOPA treatment. These results suggest a role for M1 neurons in the development of LID [6]. In humans, although M1 volume was significantly negatively correlated with the development and severity of diphasic LID, no such relationship could be proven for density; therefore only volume can be considered as a marker of diphasic dyskinesia in patients [7].

Lesioning the striatum of rats decreases the dopaminergic innervation in M1 ipsilateral to the lesion. It is represented by the loss of tyrosine hydroxylase fibers, whose density in the lesioned side is $75 \%$ lower than in the side contralateral to the lesion. Lesioning also influences the levels of M1 monoamines and their metabolites. Dopamine and norepinephrine levels are decreased, but administration of L-DOPA restores the level of DA back to the regular values [8].

A retrospective analysis of 484 patients showed an association between WMHs and LID in patients with PD. The results showed that periventricular, lobar, basal ganglia and infratentorial hyperintensities were associated with the occurrence of dyskinesia. 
Although such a result provides evidence of an association between lesions and LID, it also indicates that any WMHs location is not dominant in influencing the development of LID [9].

\subsubsection{Basal ganglia}

Neurons of basal ganglia: the internal globus pallidus (GPi) and subthalamic nucleus (STN), present an altered firing rate in dyskinetic patients. Both grouped discharges, as well as low frequency firing are significantly more frequently found in this group. Chronic treatment with L-DOPA alters activity of basal ganglia without changing the striatal DA release $[10,11]$. It also increases interactions between STN and entopeduncular nucleus, which in rodents is a structure analogous to human GPi [13]. Therefore, it is possible, that changes in firing pattern of basal neurons contribute to development of LID [10,11]. Although electrophysiological parameters of STN are not associated with the degree of severity of AIMs, attenuation of STN rebalances activity of D1R and D2R signalling pathways, which results in decreased severity of LID [12].

\subsubsection{Cerebellum}

Cerebellar circuits are specifically addressed in studies of LID development. Functional connectivity (FC) of the cerebellum and other parts of the brain at rest was studied in groups of PD patients without LID, PD patients with LID, and controls. The functional connectivity between the motor cerebellum and posterior cortical and cerebellum in PD patients is higher than in non-PD patients. The presence of LIDs is positively correlated with an increase in FC between lobule VIIIb and the left inferior frontal gyrus [14]. Cerebellar involvement in dyskinesia is also associated with red nucleus (RN) iron levels. This parameter is elevated in PD patients with dyskinesia, whereas it remains unchanged in PD patients without dyskinesias. Because the RN is involved in cerebellar circuits it is thought that iron level in RN reflects increased activity of cerebellar pathways. Their increased activity may be a compensating mechanism in response to the development of LID [15].

\subsubsection{Circulation and angiogenesis}

Local cerebral blood flow $(\mathrm{CBF})$ is increased under hypercapnia in areas of vascular proliferation. D1R activity stimulates vasodilatation and angiogenesis [16]. Abnormally elevated levels of putaminal cerebral glucose metabolism rate (CMR) are observed during an OFF phase in both dyskinetic and non-dyskinetic patients. These high CMR values decrease after administration of L-DOPA. It also causes an increase in putaminal CBF values in $75 \%$ of dyskinetic PD patients and in $50 \%$ of non-dyskinetic PD patients. The difference between the two groups of patients, although it exists, is not significant enough; therefore, neither CBF nor CMR can be considered as an indicator of changes leading to the development of LID in patients with PD. On the other hand, the parameter that significantly differentiates dyskinetic and non-dyskinetic patients is the resting activity of the primary somatosensory cortex and M1. In addition, the topography of dyskinetic symptoms reflects cortical areas that show abnormal CBF and CMR baseline values [17]. Although there is no significant difference in CBF and CMR values, these parameters can be further used to determine the putaminal hyper-perfusion/hypometabolism index (PHI). It compares ON-phase putaminal perfusion and glucose metabolism with values measured for thalamus, which is a structure similar in size to putamen that is not affected by D1R activity. PHI reflects abnormal activity of putamen after administration of LDOPA. The presence of LID in PD patients is associated with higher PHI values. It is possible, that this parameter can be used as a marker to assess the risk of developing LID in patients with PD [18].

\subsubsection{Alterations in cerebrospinal fluid}

Cerebrospinal fluid (CSF) analysis is one of the basic neurological examinations, providing a very useful insight into the CNS state. Significant differences in DA and NA turnover rate in CSF are observed between dyskinetic and non-dyskinetic PD patients (higher in the dyskinetic group). In PD patients not treated with L-DOPA, a decrease in 
CSF concentrations of the metabolites: dihydroxyphenylacetic acid (DOPAC) and homovanillic acid is observed. Physiologically, DA concentrations increase as the age-dependent DOPAC/DA ratio decreases. These observations suggest DA in CSF, as well as the concentrations of their metabolites, are potential prognostic markers for the development of LID [19], although they need confirmation in future studies.

\subsubsection{Metabolic changes}

DA deficiency results in impaired metabolism of neurotransmitters - mainly glutamate (Glu), glutamine, and aspartate in specific brain regions: midbrain and right cortex. Parkinsonian and dyskinetic rats show lower levels of synaptophysin compared to control animals, which may indicate reduced synaptic plasticity. Changes in astrocyte activity, resulting from elevated myo-inositol levels, may also contribute to development of dyskinesia [20].

\subsection{Genetic background}

The latest technologies offered by genetics and molecular science have been used to study the genetic determinants of dyskinesias in Parkinson's disease. Single nucleotide polymorphism (SNP) in GALNT14 (2p23.1; MIM 608225) gene (rs144125291), as well as in gene for GRIN2A subunit of NMDA receptor (rs7192557 and rs8057394) are significantly correlated with LID. GALNT14 encodes information concerning one of the Golgi proteins involved in glycosylation. It may be therefore involved in the basal levels of neuroinflammation in brain [21,22]. The MAOB gene polymorphism (MIM 309860) - rs1799836, G allele - is associated with increased risk of LID development, when compared to A allele [23]. Levels of long non-coding RNA (lncRNA) molecules, such as NONRATT023402, are altered in dyskinetic organisms. The regulatory targets of this lncRNA molecule are Gsto2 (10q25.1; MIM 612314) and Ptger3 (1p31.1; MIM 176806) genes, which are associated with PD and LID [24].

As the DA levels decrease during PD, gene expression is altered in spiny projection neurons (SPNs) of the direct (dSPNs) and indirect (iSPNs) pathways. These changes are particularly evident in genes regulating MAPK signalling. The difference between dSPNs and iSPNs in level of expression of Ap-1, ERK and cyclic AMP-responsive element binding protein - dependent genes is clear with more changes occurring in dSPNs. Cellular homeostatic mechanisms counteract these changes, but without effect on Ap-1-dependent gene expression. Genes associated with development of AIMs - Sstr2 (17q25.1; MIM 182452), Sstr4 (20p11.21; MIM 182454), and Kcnn3 (1q21.3; MIM 602983) - are overexpressed in dSPNs [25].

Chronic L-DOPA treatment leads to changes in DNA methylation activity, by increasing in the expression levels of genes for DNA demethylases, Tet3 (2p13.1; MIM 613555) and Gadd45b (19p13.3; MIM 604948). These epigenetic changes correlate with downregulation of other genes expression, as a result of prolonged L-DOPA administration. They are also associated with increased expression of genes involved in synaptic plasticity [26]. Bromodomain and extraterminal family proteins bind to acetylated histones, thus playing an effector role in transcriptional regulation. Their dysfunction has been associated with reorganization of dorsal striatal chromatin and with changes in corticostriatal plasticity [27].

\subsection{Alterations of neurotransmission}

Degeneration of presynaptic neurons can be measured by single-photon emission computed tomography (SPECT) of the dopamine transporter (DAT). A measure of this process is the decrease in specific binding ratio (SBR) of ${ }^{123}$ I-FP-CIT. In patients with LID, presynaptic degeneration is significantly more severe than in patients without dyskinesias, whereas postsynaptic dopaminergic neurons are preserved. This imbalance is thought to contribute to development of LID [28]. Repeated measurement (0, 24 and 48 months) of putaminal SBRs is a valid method to assess the risk of developing LID in PD patients. It shows a significant ROC curve score for prediction of LID and is associated 
with Movement Disorder Society - Unified Parkinson's Disease Rating Scale (MDS-UPDRS) Part II score [29].

Using functional MRI technique, it is possible to investigate which connections between the cortex and striatum are modulated by chronic L-DOPA treatment and evaluate the effect of dopaminergic modulation of connections on the severity of LID in patients with established dyskinesia, as well as in non-dyskinetic PD patients. An acute dose of LDOPA triggers changes in dopaminergic modulation (via feedback connections from the putamen to M1 and presupplementary area (preSMA)), although the extent of the provoked response differed significantly between patients with and without LID. Increased signalling between the putamen and M1 is thought to account for the inadequate 'Go' response, observed in NoGo trials [30]. No-Go activity in preSMA has also been shown to be a predictive measure for severity of dyskinesia. Given the overactivity of putamen in LID, this structure may be a key factor in development of dyskinesia [31].

Dyskinesia can be induced without administration of L-DOPA - by the reactivation of striatal neurons. Inhibition of their activity thus leads to a lack of dyskinetic response to L-DOPA. Neurons in striatum are mainly SPNs, but the $\gamma$-aminobutyric acid (GABA)ergic fast spiking interneurons (FSIs) and iSPNs are also present in this brain region [32]. The activity of dSPNs decreases with dopamine depletion in the midbrain, resulting in disproportion in the activity of dSPNs and iSPNs. After administration of L-DOPA, the activity of dSPNs increases again, while the activity of iSPNs decreases, leading to a restoration of balance. In LID, the effect of L-DOPA on the activity of both SPN populations is enhanced $[33,34]$. Activation of dSPNs alone is sufficient to induce LID, as demonstrated by laser light provocation. Both selective D1R and D2R agonists activate a comparable number of striatal neurons and both induce LID, although selective D1 agonists trigger more vigorous changes in glow rate and more severe dyskinetic symptoms [33]. Interestingly, the antiparkinsonian effect of L-DOPA is also mediated to a more extent by D1R than D2R [35]. The activity patterns of SPNs are altered in PD and LIDs. Dopamine depletion is associated with increased burst spike frequency or pause loss. Due to the complexity of interactions between dopaminergic and glutamatergic systems, the exact mechanism leading to these changes remains unclear. As mentioned earlier, dopaminergic signalling provokes a response that is different for the SNP subpopulations resulting in an imbalance of their activity [36].

The striatum and cortex of parkinsonian brain are bidirectionally connected. As the disease enters an advanced stage, these connections change their characteristics. It is unclear, whether the connections become unidirectional, or whether the observed imbalances are due to increased strength of one direction relative to the other. Dyskinetic events are associated with a frequency peak of $80 \mathrm{~Hz}$, in both animal model and humans $[37,38,39,40]$. Long-term potentiation (LTP) and long-term depression (LTD) are mechanisms that contribute to synaptic plasticity. Long-term potentiation occurs in indirect pathways in PD patients and in direct pathways during dyskinesias, whereas LTD occurs in direct pathways in PD patients and in indirect pathways during dyskinesias. Thus, dyskinesia is not triggered by impaired LTP induction, but results from a reduced excitability difference between dSPNs and iSPNs during ON-phase [40,41,42].

\subsubsection{Inhibitors of monoamine oxidase B and catechol-O-methyltransferase}

Both of these enzymes are one of the key components of DA metabolism. Their inhibitors are widely used as adjuvant therapy in the treatment of PD with L-DOPA [43]. Identification of SNPs of genes for monoamine oxidase B (MAO-B) and catechol-O-methyltransferase was thought to be useful in estimation of activity of these enzymes, which in turn influences DA metabolism and indirectly dyskinetic symptoms [44,45].

\subsubsection{Dopaminergic pathways and dopamine receptors}

Three distinct signalling pathways - PKA/DARPP-32, ERK and mTORC1 play an important role in the mechanism of LID development. They are all activated by a common intracellular cascade, that is triggered in striatonigral SPNs expressing D1Rs. Long-term L-DOPA administration increases the activity in these pathways, leading to the changes 
in striatal bidirectional synaptic plasticity. Activation of one pathway may affect the activity of other pathways. DARPP-32 signalling modulates ERK and mTORC1 pathways $[46,47]$

Recent studies have closely examined the effect of D1R activation on ERK1/2 through interaction with the tyrosine phosphatase Shp-2. Not only has Shp-2 activation been shown to be a key factor for ERK1/2 and mTOR activation via phosphorylation and consequently for LID expression, but it has also been demonstrated that long-term stimulation with intermittent L-DOPA or D1R agonists leads to Shp-2 phosphorylation. This effect can be counteracted by D1R antagonists, consequently influencing the levels of the D1R/Shp-2 pathway end products - p-mTOR and p-ERK1/2 [48]. Rapamycin was shown to selectively affect mTORC1 in the striatum without affecting either PKA/DARPP-32 and ERK pathways or the expression of $\alpha$-amino-3-hydroxy-5-methyl-4-isoxazolepropionic acid receptor (AMPA) and NMDA receptor subunits [47].

In lesioned animal models, the potential of D1R to associate with PKA and ERK1/2 was increased, and this change occurred concomitantly with the onset of LID. However, within 2 weeks after the treatment with L-DOPA, D1R were not able to activate PKA and ERK1/2 pathways. These observations indicate a remodelling of direct striatal pathways, that results from oversensitivity of dSPNs [49]. The rate of D1R-dependent ERK1/2 phosphorylation is modulated by mGluR5, which alters mGluR5/PLC/PKC pathway through modulation of calcium/dependent responses. However, it does not influence the PKA activity [50]. Hypersensitivity of D1R/cAMP/PKA pathway is associated with the elevation of $\mathrm{G} \alpha$ olf protein level. This protein is the major striatal stimulating G-protein, whose activity leads to increased cAMP levels. Interestingly, despite the decreased $\mathrm{G} \alpha$ olf protein levels in mutant animals, the D1R/cAMP/PKA pathway was found to be hypersensitive in these subjects. Therefore, the activity of $\mathrm{G} \alpha$ olf may not be essential for hypersensitivity of this pathway [51]. Chronic L-DOPA administration is associated with increased levels of G $\alpha_{\text {olf }}$ protein in striatonigral SPNs, and decreased level of this protein in striatopallidal SPNs [52].

Knocking-out casein kinase 2 (CK2) is also associated with reduced Golf levels in striatonigral SPNs. Knockout (KO) of CK2 in striatonigral neurons in model animals results in decreased severity of dyskinesia. This also correlates with lower pERK levels in dSPNs and cholinergic aspiny interneurons (ChIs). When CK2 is knocked-out in striatopallidal neurons, the severity of dyskinesia and pERK levels in dSPNs and ChIs increase. However, in the latter case the LID can be attenuated by coadministration of caffeine with LDOPA [53].

Increased expression of Nurr1 transcription factor in striatal neurons of dyskinetic rats is associated with increased cortically-evoked firing rate and increased spine density of SNPs [54]. Both downstream D1R/D2R activation pathways, PKA and ERK1/2, are influenced by activity of leucine-rich repeat kinase 2 (LRRK2). The interactions between these pathways and LRRK2 are not yet well understood, but their further investigation may result in a new therapeutic strategy to modulate the downstream activity of dopaminergic receptors, leading to a better control of LID [55]. The dopaminergic system in midbrain is also regulated by activity of vesicular glutamate transporter type 3 in circadian-dependant matter [56].

Surface distribution of dopaminergic receptors is dictated by anchoring protein postsynaptic density protein 95 (PSD95). Its levels are increased in dopamine-depleted brains, causing lower diffusion of D1R in the membrane. Association between DA loss and increased levels of PSD95 might be involved in the development of dyskinesia [57]. Activity of parkin, an E3 ubiquitin-protein ligase, is impaired not only in genetic parkinsonism, but also in sporadic PD patients. Loss of function of this enzyme leads to sequestration of ubiquitinated proteins. It is associated with D1R-mediated decreased proteasome catalytic activity, which is reflected by severity of AIMs [58].

$\mathrm{Ca}+/$ calmodulin-dependent protein kinase II $\alpha$ (CaMKII $\alpha$ ) binds to the intracellular domain of D2R. The Gai domain of D2R is also localised in this region. This may indicate that CaMKII $\alpha$ affects D2R activity in adenylyl cyclase signalling pathway. In parkinsonian 
rats, The level of interaction between CaMKII $\alpha$ and D2R is elevated in parkinsonian rats. Decreasing its level leads to attenuation of LID with efficacy similar to D2R agonists [59].

An increased D3R response is observed in animals with severe dyskinesia, which is associated with increased GABA release. This differs significantly from animals with lower AIMs scores. D3R can affect ERK pathway independently of D1R, thus adding up to the effect of D1R modulation [60]. Deletion of D3R is associated with decreased levels of FosB, ERK and $\mathrm{H} 3$ activity. It also causes attenuation of dyskinesia, with preservation of the therapeutic effect of L-DOPA [61]. Main locus of D3R, whose levels are elevated in dyskinetic patients, is the GP [62]. Lack of D5 signalling in D5R animals is associated with decreased therapeutic effect of L-DOPA and increased expression of LID. As LID increases, LTD plasticity is reduced in these animals. Decreased acetylcholine (ACh) release also negatively affects the activation of M1 postsynaptic receptors in dSPNs [63].

\subsubsection{GTPases of Ras family}

Raf/Mek/ERK is a pathway that activates the ERK signalling cascade. It is stimulated by Ras GTPases, whose activity is increased by DA and glutamate receptors. Decreasing the striatal levels of Ras-guanine nucleotide-releasing factor (Ras-GFR1) leads to inhibition of Ras/ERK pathway, which might be an important management strategy in LID. RasGFR1 affects the Ras-ERK pathway only in dSPNs, without affecting cholinergic interneurons of ChIs [64]. It therefore affects specific downstream D1R pathways, consequently inhibiting the development of hypersensitivity to DA stimulation in the neurons. This is associated with less development of LID, although dopaminergic stimulation retains its therapeutic effect on parkinsonian motor symptoms.

Administration of drugs that modify the MEK-ERK core components further potentiates the aforementioned antidyskinetic effect. Elimination of Ras-GRF1 decreases ERK, PKA and mTOR signalling intensity $[65,66]$. Consequently Ras-GRF1 levels in striatonigral SPNs also affect LTP-dependent synaptic plasticity. Such a relationship was not observed in striatopallidal SPNs, which may be due to the involvement of DARPP-32 or Ras-GRF2 activity [67]. The Ras-ERK pathway in mice can be overactive due to overexpression of Ras-GRF1, or due to reduced gene expression for Ras inhibitor (Nf+/-). Interestingly, in both cases the severity of dyskinesia does not exceed normal levels. However, administration of lovastatin, a Ras inhibitor, improves control of dyskinetic symptoms only in the group with normal Nf+/- gene expression [68].

\subsubsection{FosB transcription factor}

Chronic treatment with L-DOPA leads to increased level of truncated splice variant of FosB $(\triangle \mathrm{FosB})$. Among others, This transcription factor regulates genes responsible for plasticity in striatal neurons. Elevated levels of $\Delta$ FosB are associated with loss of function of FosB, which results in early development of dyskinesia and unstable firing rate changes during ON phase in L-DOPA treated animals [69]. MSH1 histone kinase activity also increases with $\triangle$ FosB levels, consistent with MSK1's target, histone H3. H3 is localised on cfos-associated nucleosomes, and its phosphorylation results in increased expression of this gene [64]. In SPNs expressing D1R, MSK1 also decreases Polycomb-group-proteinsdependent gene repression [70].

The level of c-Fos expression in M1 neurons differs between sides in an animal model of unilateral medial forebrain bundle injury after acute $(p=0.003)$ or chronic $(p=0.001) \mathrm{L}$ DOPA treatment, although it is elevated, when compared to the control. It is also positively correlated with total AIMs score $(p=0.13)$. Another immediate-early gene product, ARC, which is associated with synaptic plasticity, showed no such correlation [71]. Both of these genes are overexpressed in dorsolateral bed nucleus of the terminal stria (dlBST) in dyskinetic rats. This structure is not included in the basal ganglia, and as such has not been implicated in the development of LID. Interestingly, inhibition of $\triangle F$ FosB expression in dlBST results in reduced AIMs score [72]. The neuronal NO synthase inhibitor 7-nitroindazole also downregulates $\mathrm{FosB} / \Delta \mathrm{FosB}$ expression and reduces dyskinetic symptoms [73]. 


\subsubsection{Glutamatergic signalling}

Neurotransmission systems other than dopaminergic also affect SPNs and contribute to the development and expression of LID. One of these is glutamatergic system. As the duration of L-DOPA therapy increases, NMDAR activity and their composition changes. Interactions of Rph3A protein with the GluN2A subunit of NMDAR's are increased in parkinsonian dyskinetic rats. Altering of PSD95 function leads to improved AIMs scores in animals. This may be a promising target for antidyskinetic therapy, despite potential post-synaptic interactions involving PSD95 [74]. This protein also contributes to overactivity of NMDAR in dyskinetic animals. It modulates interactions between Fyn kinase and NR2B subunit [75]. AMPA antagonists directly acting on glutamate receptors in M1 are effective in reducing the severity of dyskinesia. NMDA antagonists did not alter dyskinesia in parkinsonian rats, although simultaneous action on NMDA and AMPA receptors increased the effect of AMPA antagonism [76].

\subsubsection{Cholinergic signalling}

The density of nicotine cholinergic receptors (nAChRs) in the striatum of dyskinetic PD patients remains normal, in contrast to the reduced levels of $n A C h R s$ in non-dyskinetic patients. This indicates that cholinergic activity is preserved in LID, which may be a mechanism of overcoming DA loss in striatal neurons. Cholinergic activity induces DA release from otherwise serotoninergic neurons. Regulatory mechanisms of dopaminergic transmission do not affect the above DA release, as evidenced by the lack of significant differences in the measurement of DAT binding in the striatum between dyskinetic and nondyskinetic patients [77].

Metabotropic muscarinic cholinergic receptors (mAChRs), specifically M1R and M4R are involved in LID expression. Interestingly, M4R has a bidirectional function - endogenous ACh inhibits LID and striato-nigral SPN activation via M4R stimulation, wherease during LID it facilitates its effect on postsynaptic M4 mAChRs. Exogenous stimulation of mAChRs in M4, presumably at presynaptic dopaminergic terminals or ChIs, also potentiates inhibitory effect on LID [78]. Activation of M4Rs by endogenous ACh potentiates LTD and inhibits LTP in dSPN glutamatergic synapses. Thus, the function of M4Rs is similar to that of D2R in iSPN. Exogenous stimulation of M4R increases synaptic plasticity in dSPNs and thereby attenuates LID [79].

\subsubsection{Serotonergic signalling}

Serotonergic neurons are capable of converting 5-HT into DA, storing the produced DA and then releasing it. This function is referred to as the presynaptic serotonergic LID mechanism. The observed greater severity of dyskinesia in animals with an abundance of serotonergic endings in the striatum is not due to DA release per se, but rather to an abnormal activation pattern (with a predominance of D1R over D2R) and lack of appropriate DA gradient [80]. In dyskinetic rats, selective serotonin reuptake inhibitors (SSRIs) such as citalopram, paroxetine and fluoxetine show a dose-dependent antidyskinetic effect when administered together with L-DOPA. In contrast, no such effect was observed in animals treated with apomorphine, a direct DA agonist. This indicates a presynaptic mechanism of antidyskinetic action [81]. L-DOPA administration does not affect 5-HT receptor activity, although it reduces the effect of SSRI on neuron firing rate in dorsal raphe nucleus (DRN) [82].

\subsection{Clinical and environmental background}

Certain non-modifiable factors and clinical features are associated with earlier onset or higher severity of LID. Female gender is an independent risk factor for developing LID. Patients whose tremor predominates over rigidity and akinesia have been shown to have a lower risk of LID [3], but these data are inconclusive. In another study, patients who initially had tremor had a significantly higher risk of LID during follow-up [83]. The course of treatment also influences the characteristics of LID in PD. A higher mean dose of L-DOPA during the first 6 months of therapy, as well as greater severity of motor symptoms, are significantly associated with early onset of LID. These observations are mutually 
consistent - patients who initially have more severe parkinsonian motor symptoms require higher doses of L-DOPA to restore normal motor function. Moreover, the need for more rapid escalation of the drug dose is a direct reflection of the rapid degeneration of the dopaminergic system and correlates with earlier onset of LID. It has been pointed out, however, that it is not clear whether L-DOPA administration is a contributing factor to the development of LID itself, or rather just a trigger [3,84,85]. Nonetheless, less-pulsatile administration of L-DOPA, e.g. 6x doses per day, has been shown in clinical trials to reduce the risk of LID development in both: in PD patients who have never previously initiated L-DOPA treatment and in non-dyskinetic patients treated with L-DOPA [86]. A continuous supply of L-DOPA via an enteral levodopa-carbidopa gel reduces fluctuations in DA levels. In patients with dyskinetic symptoms lasting more than $4 \mathrm{~h} /$ day, a reduction in LID duration, severity and accompanying pain was observed after administration of levodopa-carbidopa enteral gel. In patients with duration of dyskinetic episodes less than $4 \mathrm{~h} /$ day, the duration of dyskinesias increased, albeit with a significant reduction in severity and pain [87].

Adenosine A2A receptor antagonists may reduce the severity of dyskinesia, which is associated with greater receptor availability in PD patients who develop LID [88]. Caffein, as an A2A antagonist, may be a factor delaying the onset of LID [89]. The N-of-1 study on nicotine use demonstrated a significant effect of nicotine on the occurrence of LID compared to placebo [90].

\subsection{Pharmacological treatment}

\subsubsection{Modification of L-DOPA delivery and release}

Because LIDs are motor symptoms caused by pulsatile changes in DA concentration due to intermittent L-dopa administration, the obvious strategy to prevent them is continuous delivery and release of the drug (continuous dopamine stimulation; CDS) - this way, theoretically, DA concentration will reach a stable level at which the therapeutic, antiparkinsonian function is active without LID side effects.

L-DOPA/benserazide poly(lactic-glucosic acid) (LBM) microspheres are a novel therapeutic option for the treatment of PD. In animals, LBM showed a dose-dependent antiparkinsonian effect and did not significantly increase AIMs scores. In control animals treated with L-DOPA, D1R/Shp-2/ERK1/2 pathway activity increased with duration and onset of dyskinesia, which was not observed in LBM-treated animals [91]. These results are consistent with a previous study that also demonstrated the efficacy of LBM therapy in reducing LIDs frequency [92]. Abnormal PKA/DARPP-32 signalling leads to increased phosphorylation of tau protein, which in turn activates SPNs and results in LID. PKA/DARPP-32 and tau protein phosphorylation activity was increased in mice with parkinsonism treated with standard i.p. L-DOPA/benserazide (LS) therapy. A dose-dependent inhibition of PKA/DARPP-32 and tau protein phosphorylation was observed in LBMtreated animals [93].

Striatal PKA-related glutamatergic signalling and glutamatergic receptors (AMPA) may play an important role in the expression and occurrence of LIDs. During chronic repeated L-DOPA treatment, the AMPA receptor subunit, GluR1, becomes over-phosphorylated at the serine-831 and serine-845 position. Such changes (elevated levels of pGluR1S831 and pGluR1S845 ) are not found in LBM -treated animals [94].

An alternative method of L-DOPA delivery is chitosan-coated nanoliposomes (CCN). ERK1/2, DARPP-32, and FosB/ $\triangle$ FosB expression and LID induction are significantly less elevated in parkinsonian rats treated with $\mathrm{CCN}$, compared to the same animal model treated with LS. However, these parameters in PD rats treated with CCN are higher than in control group of saline-treated animals [95].

An extended-release (ER) L-DOPA/carbidopa (CD-LD) (product name: IPX066) prolongs the duration of ON phase without dyskinesia, shortens the duration of OFF phase, and does not increase the severity of LIDs, compared with the treatment effects of immediate release (IR) CD-LD, and CD-LD with entacapone. Concomitant treatment with CD- 
LD and DA agonists, MAO-B inhibitors, or amantadine does not significantly affect treatment outcomes, although small changes in treatment efficacy have been observed [96,97].

\subsubsection{Long-releasing amantadine counteracts LID}

Amantadine, an antiparkinsonian drug, is used to reduce LID. In a study conducted in an animal model, administration of amantadine decreased the severity of LID episodes. Interestingly, GABA release in SN, which was increased after L-DOPA treatment and during AIMs, increased significantly less when L-DOPA was administered concomitantly with amantadine. This suggested that the mechanism of antidyskinetic effect of amantadine may be related to an effect on nigral GABA levels [98]. Recent studies have shown that, amantadine inhibits dyskinesia by blocking of inward-rectifying type $2 \mathrm{~K}+$ channel in SPNs rather than NMDA receptors [42].

Extended-release amantadine (ERA) capsules (product name: ADS-5102) significantly $(27 \% ; p=0.005)$ reduce dyskinesia and increase the duration of drug efficacy without dyskinesias in the experimental group compared to control group [99]. ERA has also been shown to improve MDS-UPDRS [100,101]. Adversary effects (AEs) of long-term ERA therapy were dose-dependent and most commonly included falls, sleep disorders and hallucinations. In most cases they were not caused by the drug itself, but were due to the severity of PD course. NMDA-receptor antagonism caused hallucinations, and cholinergic antagonism caused symptoms such as dry mouth, constipation, and nausea [99,102,103]

\subsubsection{D3 selective agonism}

Another suggested solution for LID is to rebalance the function in D1 and D3 receptors - both receptors are co-expressed in dSPN, and studies in animal models have shown that D3 agonists are able to reduce the incidence of LID [104,105]. Pramipexole is a nonergoline agonist that prefers D3 receptors. Pramipexol monotherapy (achieved by gradually replacing previously used drugs with pramipexole) led to significant decrease in Core Assessment Program for Surgical Intervention Therapies scores compared with baseline values after 4 weeks of the treatment and was maintained at this level. Patients treated with pramipexole had significant improvement of MDS-UPDRS part III (improvement in motor symptoms) and quality-of-life after 2 weeks of therapy. The only observed AEs were increased dyskinesia and prolonged duration of the OFF-phase. D3 agonism is a potential candidate for reducing LID in clinical practice because it combines the efficacy of PD treatment, LID control, and safety of use. This may be due to the synergic effect of D1 and D3. Agonists preferring D3 may provide stimulation levels to overcome the hypokinetic symptoms of PD, but also low enough to not induce LID [106].

A new drug (product name: IRL790), selective D3 receptor antagonist, has been proposed. Dyskinetic animals show increased D3R expression in striatum [107], so D3 receptor antagonism may be a potential strategy for reducing dyskinesia. D3 receptor antagonists, partial agonists, and D3R deletion attenuated the occurrence of LID [104]. Treatment caused a significant number of AEs, with $90.1 \%$ of patients taking D3-selective antagonists experiencing at least one AE, which was less than the AEs in placebo group. The most common AE in the IRL790 group was worsening parkinsonism. Significantly more AEs were observed during dose titration of the drug than during the dose-size stabilization phase. Treatment of efficacy was measured using Unified Dyskinesia Rating Scale, showing a median reduction of 11,5 points and a mean reduction of 8,2 points in the intervention group compared to placebo. The drug has been shown to be able to significantly reduce LID, although the concomitant presence of AEs must also be taken into account. [108].

3.5.4. Multile handle-point strategy - safinamide

This relatively new drug stands out because it combines both dopaminergic (increased DA reuptake) and non-dopaminergic (MAO-B inhibition sodium channels blockade, stimulation of Glu release) mechanisms of action. Safinamide prolonged the ON phase duration without dyskinesias or with non-obstructive dyskinesia, and shortened OFF phase. The improvement was significant for both higher and lower doses of 
safinamide in comparison with placebo, although the higher dose of safinamide showed greater efficacy. No increase in the severity of dyskinesia was observed [109]. Long-term safinamade therapy improved scores on the MDS-UPDRS-II, -III, -IV; CGI-C; PDQ-39 and GRID-HAM-D scales. The following AEs occurred in more than $10 \%$ of patients: cataract, asthenia, pyrexia, fall, back pain, dyskinesia, PD worsening, headache and insomnia. After 18 months of treatment, the frequency and severity of new dyskinesias were similar between experimental and placebo groups [110]. Post hoc analysis confirmed these results [111]. In rats, safinamide did not prevent the development of LID or activation of the direct dopaminergic pathway. Increased levels of Glu in the striatum, which is associated with dyskinesias, were not observed in safinamide-treated rats [112].

\subsubsection{Serotonin in the treatment of LID}

The serotoninergic system is largely present in the basal ganglia. Chronic L-DOPA treatment and decreased DA concentration leads to its alterations. It is speculated that drugs that modify 5-HT activity may have an inhibitory effect on LID. However, they require careful administration because overdose of serotonin agonists leads to 5-HT syndrome, symptomatology similar (tremor and rigidity) to PD [113]. The 5-HT1A partial agonist buspirone inhibits LID by influencing the 5-HT1A receptors, although it does not alter STN electrical activity [114]. It also regulates GABA and Glu release and burst activity in pars reticular of $\mathrm{SN}(\mathrm{SNr})$ in rats [115].

The 5-HT1A agonist 8-hydroxy-2-dipropylaminotetralin (8-OH-DPAT) significantly reduces LID, but this benefit is outweighed by the 5-HT syndrome that is caused by 8 OH-DPAT. Nonetheless, 8-OH-DPAT delays LID development [116] and reduces elevated cortical gamma activity, that was caused by D1 and D2 agonism [117]. The efficacy of SSRI (citalopram) in in inhibiting LID is similar to 8-OH-DPAT and buspirone, but it does not induce significant 5-HT syndrome [118]. LID inhibition by another 5-HT1A agonist, BMY-14802, increases adequately with the dose $(20>10>5 \mathrm{mg} / \mathrm{kg})$. The antidyskinetic effect of BMY-14802 was observed not only in LID induced by L-DOPA administration, but also in LID induced by the administration of D1 and D2 agonists. However, this effect can be reversed by using 5-HT1A receptor antagonists in L-DOPA induced LID [119].

Treatment with 5-HT1A/B agonist, eltoprazine, Clinical Dyskinesia Rating Scale scores were significantly reduced at the $5.0 \mathrm{mg}$ and $7.5 \mathrm{mg}$ dose, although significant reduction in maximum LID severity was observed only at the $5.0 \mathrm{mg}$ dose. No significant changes in MDS-UPDRS-III scale scores were observed. While the above therapeutic results are not as spectacular as those previously reported in animal models, such a difference was to be expected given the differences in the complexity of the substrate of LID in humans and rodents [120].

The 5-HT3 antagonism was another serotoninergic system checkpoint that was investigated for its potential to reduce LID. In rats the first significant differences in AIMs scores were observed after 9 days of treatment with ondansetron (5-HT3 antagonist) + LDOPA, compared with animals treated with L-DOPA alone. This difference steadily increase. Interestingly, ondansetron administration was discontinued on day 23, resulting in significant differences between the L-DOPA + ondansetron and control groups on day 30. These results indicate the potential of 5-HT3 antagonists such as ondansetron, in controlling LID in the treatment of PD [121].

3.5.6. $\beta$-adrenoceptor blockade - propranolol

To date, the modulation of LID by adrenergic compounds has emphasized the $\alpha$ adrenergic receptor $(\alpha \mathrm{AR})$, despite the high density of $\beta$-adrenergic receptors $(\beta A R)$ in the striatum of PD patients. The optical isomer (-) of propranolol affects $\beta$ ARs in dorsal striatum and is the isomer responsible for reducing LID, with 2-fold longer antidyskinetic effect compared with $( \pm)$ compounds. Although propranolol alone can reduce the motor activity of rats, which is highly undesirable in the treatment of PD, there is a broad doses spectrum in which this reduction does not occur, with a concomitant significant effect on LID. Supportive therapy with propranolol normalizes aberrant signalling in the striatum 
[122]. Propranolol could not attenuate LID induced by D1 and D2 receptors agonists, indicating that propranolol acts through a presynaptic mechanism [123]. Because of the association between reduction of striatal DA, propranolol administration and the reduction of LID reduction, it was suggested that the antidyskinetic mechanism of propranolol may be related to the reduction of striatal DA levels. It may be related to the secondary anticholinergic effect of propranolol. Inhibition of $\beta$ ARs results in decrease intracellular cAMP, which leads to a decrease in the spontaneous firing rate of ChIs, resulting in a decrease in ACh levels. Striatal DA release is regulated by ACh, and thus the effect of propranolol on ACh levels would restore the balance between ACh and DA in LID [124].

3.5.7. Nicotinic receptor and its agonists

Acetylcholine receptors (AChR) of nigrostratial neurons modulate neurotransmission, including dopaminergic signalling. Nicotine, a direct agonist of $\mathrm{nAChR}$, has been shown to significantly reduce AIMs scores after $8(p<0.01)$ and $12(p<0.05)$ weeks of administration. Although acute nicotine administration stimulates $\mathrm{nAChR}$, thereby increasing dopamine release [125], intermittent nicotine treatment desensitizes nAChR. It also decreases dopamine release, which may explain its antidyskinetic effect - in later stages of PD, DAT levels are reduced, resulting in less efficient clearance of excess DA [126]. Interestingly, a more recent experiment showed no significant improvement in dyskinesia in female mice after 10-week treatment of nicotine, as well as in the group treated for 5 days with $\alpha 7 \mathrm{nAChR}$ partial agonist (product name: AZD0328). Brain-derived neurotrophic factor (BDNF) levels in the striatum, but not in the prefrontal cortex, were shown to be significantly correlated with development of LID. Nicotine failed to significantly reduce high BDNF levels in the striatum, but succeeded at the prefrontal cortex level. It was suggested that the reason for the lack modulation of LID by the drugs tested is due to the condition of the animals - their nigrostriatal damage was almost complete, indicating that treatment of LID with nicotine requires less damaged nigrostriatal pathway [127].

\subsubsection{Ionotropic receptors of Glu}

The previously described increase in glutamatergic transmission in the development of PD and LID indicates that its inhibition appears to be an attractive therapeutic approach for the treatment of LID. As the disease progresses, an increase in the NMDA subunits, NMDA-R1 and NMDA-R2, acting as an ion channel and regulatory unit, respectively becomes apparent. Studies on Compound Formula Rehmannia showed its inhibitory effect on NMDA-R1 and NMDA-R2 expression. It also increased the expression of GABA-RB1, which becomes down-regulated during LID. This effect was correlated with the decrease of LID in test animals [128]. Memantine, a non-competitive NMDAR antagonist, exhibits a similar mechanism through which it attenuates LID [129].

\subsubsection{Histamine $\mathrm{H}_{2}$ receptor antagonism}

Given the widespread distribution of $\mathrm{H}_{2}$ receptors in the basal ganglia involved in the development of LID, both in their input and output regions, it is conceivable that $\mathrm{H}_{2}$ receptors may be targets for antidyskinetic therapy. Chronic treatment with ranitidine reduces LID expression in 6-OHDA rats treated with L-DOPA. A suggested mechanism of action of ranitidine to reduce dyskinesias involves inhibition of the PKA pathway in the SPNs [130]. Ranitidine inhibits striatal Glu efflux, as well as GABA release in SNr, thus affecting pre- and postsynaptic mechanisms of LID development. Pre-treatment of rats with ranitidine reduces AIMs scores, without reducing the efficacy of anti-PD treatment [131].

Although the results of ranitidine in animal models appear promising, a study with famotidine in a human model showed no significant differences between the treatment effect of famotidine (doses: 80, 120, $160 \mathrm{mg} /$ day) and placebo. No significant AEs were observed in this study, demonstrating the safety of famotidine. The lack of clinically significant results in this experiment illustrates that the complexity of LID problems in humans cannot always be adequately represented in animal models, and that optimistic 
results from studies in rats or non-human primate may not necessarily translate to human experiments [132].

\subsubsection{Opioid modulators}

Because opioids affect neurotransmission in the basal ganglia and the intensity of opioid signalling is significantly altered during PD [133], drugs that modulate this transmission system appear to be an effective alternative to standard antidyskinetic therapy. Treatment with a combination L-DOPA + nalbuphine $(\kappa$ receptor agonist and $\mu$ receptor antagonist) reduce the expression of $\triangle \mathrm{FosB}$ in the striatum, as well as other LID markers: prodynorphin, dynorphin A, Cdk5, Thr34-phosphorylation of DARPP-32, to normal values. Drug administration did not cause any AEs, and even the sedative effect present in nalbuphine monotherapy was not induced by L-DOPA administration [134]. The significant results of this experiment may be due to the dualistic nature of the treatment strategy used - highly selective $\mu$-opioid receptor antagonism did not improve dyskinesia in the tested 6-OHDA rats [135]. Only $\mu$ receptor agonists appeared to reduce the incidence of LID when used alone [136].

Nociceptin/orphanin FQ opioid peptide (NOP) receptors, despite some functional similarities, differ from opioid receptors - they are not activated by the most standard opioid receptor ligands. Although both NOP agonists, AT-390 and AT-403, exhibited dose-dependent overall hypolocomotor effects, AT-403 produced significant, albeit mild, improvement in dyskinesia [137].

\subsubsection{1. cAMP and cGMP signalling - inhibitors of phosphodiesterase 10A}

Phosphodiesterase 10A (PDE10A) is an enzyme expressed exclusively in brain tissue, including the SPNs expressing D1R and D2R. By hydrolysing cyclic nucleotides cAMP and cGMP, it affects DA signalling in SPNs, showing effect similar to D2R antagonists. In PD patients, PDE10a expression is significantly reduced in the striatum and GP. Due to the presence of PDE10A in both populations of SNPs, it is possible that its theoretical antidyskinetic effect could exceed that shown by D2R antagonists [138]. A novel PDE10A inhibitor (product name: MR1916) showed high penetration into the brain and antidyskinetic effects greater than amantadine over the entire dose range - not only with acute but also with chronic administration. It also did not cause significant AEs [139].

\subsection{Genetic treatment}

Another therapeutic approach to LID focuses on modifying the expression of LIDrelated genes. Considering the latest techniques used in molecular sciences, this area appears to be a very promising and effective strategy, and genes related to the dopaminergic system and dopaminergic pathways seem to be the first choice. In parkinsonian rats, interesting results were obtained by overexpression of DA autoreceptor D2Rs in the DRN, which is involved in previously described uncontrolled uptake and release of L-DOPA in serotonergic neurons. The DRN, in which D2Rs were overexpressed was insensitive to high, LID-inducing doses of L-DOPA and dopaminergic agonists. This demonstrated that genetic control of serotonergic DRN neurons could be used to reduce dyskinesia [140]. Inhibition of the p11 gene (1q21.3; MIM 114085) in the dorsal striatum produced surprising results. Its product, S100A10, is known to activate the serotonin 5-HT1B receptor, which in turn is associated with decreased LID. Thus, downregulating p11 expression should rather increase LID, and in fact inhibited dyskinesia more effectively than direct pharmacological activation of 5-HT1B. These results suggested that the effect of p11 on LID development functions through mechanisms not involving 5-HT1B [141]. Transplantation of dopaminergic neurons has also been investigated for the effect of L-DOPA on graft survival. Both allogeneic and xenogeneic transplants were viable, but showed no significant improvement of motor functions in the test animals. Furthermore, post-transplantation L-DOPA treatment induced an immune response in xenogeneic grafts [142]. It has been shown that ERK hypersensitivity, secondary to striatal damage in rats can be reduced by overexpression of $G$ protein-coupled receptor kinase (GRK) (2-fold). This also resulted in decreased Akt activity and decreased $\triangle$ FosB levels in striatum. Increasing GRK 
expression with lentivirus may be a potential strategy to reduce dyskinesia [143]. A direct target of GRK, a G protein-coupled receptor, is also $\beta$-arrestin2, a protein scaffolding various intracellular molecules. In rats with $\beta$-arrestin2 overexpression, a significant reduction in LID efficiency for L-DOPA was observed, without affecting the therapeutic efficacy of L-DOPA. Knocking out $\beta$-arrestin2 had the opposite effect on increasing dyskinesia $[144,145]$. Knocking out adenylyl cyclase 5 (AC5) appeared to be another option to modulate downstream dopaminergic pathways. Significant attenuation of LID was observed in AC5 KO mice, coupled with decreased activation of PKA pathway, resulting in decreased ERK level, as well as lower FosB/ $\triangle$ FosB expression [146].

Knocking out $\alpha 5$ subunit of $\mathrm{nAChR}$ in 6-OHDA-lesioned mice leads to attenuation of dyskinesia in these animals. Compared with wild-type individuals, $\mathrm{KO}$ mice showed less dopaminergic denervation in SN, as manifested by reduced rotational movements after amphetamine administration. DAT activity was also lower in modified individuals, resulting in less 6-OHDA neurotoxicity [147].

As mentioned earlier, CaMKII $\alpha$ is involved in LID expression. Modulating its function through inhibitors such as KN-93 has been shown to reduce LID. Although it did not show a significant antiparkinsonian effect, it did reduce pGluR1S845 levels and Gad1 (2q31.1; MIM 605363) and Nur77 (12q13.13; MIM 139139) gene expression, all of which were 1 increased after 3 weeks of L-DOPA treatment [148].

CaV1.3 calcium channels are present in striatal SPNs. Studies in dyskinetic animals administered nimodipine (CaV1.3 antagonist) showed a reduction in LID. Recently the effects of CaV1.3 antagonism on LID expression have been studied more directly, by silencing genes responsible for striatal CaV1.3 channels at the mRNA level. Even partial silencing of CaV1.3 in striatum led to complete prevention of LID. The method presented here was designed to specifically target the striatum, as otherwise silencing CaV1.3 in other tissues could lead to serious cardiac, neurological and psychological consequences [149].

Peroxisome proliferator-activated receptors (PPAR) have been shown to be involved in antidyskinetic effect of URB597 (an inhibitor of endocannabinoid catabolism) and capsazapine. Moreover, direct activation of PPAR $\gamma$ led to a decrease in LID, which was also correlated with an increase in ERK phosphorylation [150].

\subsection{Invasive and noninvasive brain stimulation}

Deep brain stimulation (DBS) is an invasive treatment option for advanced PD, in which the therapeutic window is increasingly narrower and pharmacological control of the disease is increasingly difficult. DBS can control both motor symptoms of parkinsonism and dyskinesias. Its primary targets are the STN and GPi. Recently, stimulation of those two regions has been shown to have different effect on LID. GPi-DBS produced a direct antidyskinetic effect, while STN-DBS affected LID mainly by reducing the equivalent daily dose of L-DOPA (LEDD) [151]. The results of retrospective analysis showed that STN-DBS improved MDS-UPDRS IV score in $57-61.9 \%$ of patients, depending on the group, with the improvement exceeding the direct effect of LEDD reduction on LID. This effect increased with time after treatment (69\% vs $77 \%$ at 1 year vs 2 years after treatment, respectively) [152]. Interestingly, stimulation of the area above STN (including the zona incerta) had better efficacy than stimulation of the STN alone (18 vs 15 patients; $\mathrm{p}=0.048$ ) [153]. Although the STN is usually used to treat advanced PD, DBS-STN was also studied in PD patients with mild motor complications and short (1.5 years) history of dyskinesia. The use of DBS-STN resulted in better treatment outcome. However, AEs were also more frequent than in the pharmacological-only treatment group [154].

Repetitive preSMA transcranial magnetic stimulation (rTMS) has been studied as an alternative, non-invasive stimulatory intervention meant to control LID. As described previously, preSMA activity is increased in dyskinetic PD patients, which may reflect pathological processes in the development of LID or attempts to compensate the pathology. The results of this study showed, that preSMA overactivity is one of the factors contributing to development of dyskinesia, and that rTMS exhibits an antidyskinetic effect 
proportional to the strength of the applied magnetic field [155]. This study confirmed the results of previous studies showing the efficacy of rTMS in neurodegenerative diseases, including PD. Low-frequency rTMS has been shown to reduce dyskinesia in PD patients, and high-frequency rTMS has been shown to improve their motor function [156].

\section{Conclusions}

Structural changes in the brain that become apparent as an increase in asymmetry in striatum size between sides, with a predominance of the degenerate side, lead to the development of LID. Functional changes in the basal ganglia appear to be central in development of LID, particularly low frequency firing. Bidirectional plasticity in cortico-striatal pathway is altered. SPNs of the direct and indirect pathway show altered frequency and pattern of firing in LID. Excitability of dSPNs and iSPNs in ON-phase is impaired. Dopaminergic downstream pathways - PKA/DARPP-32, ERK and mTORC1 are activated as part of a common cascade, beginning in striatonigral D1Rs SPNs. With the duration of LDOPA treatment, the activity of these pathways increases. D3R, located mainly in GP, shows an additive effect to that of on LID, and its deletion reduces activation of FosB, ERK and H3 . Risk factors for the development of dyskinesias include a higher mean L-DOPA dose size over 6 months, higher dose escalation rate, earlier onset of PD, greater severity of motor symptoms, and female gender. New routs of administration of L-DOPA (LBM, CCN), have been introduced, as well as ER compounds LD+CD and amantadine, with very satisfactory results in lowering LID. Selective D3R agonists, such as pramipexole, may be considered as a moderate alternative to L-DOPA. Both 5-HT-1A and 5-HT-1A/B agonists showed dose-dependent antidyskinetic effects. SSRI also reduced LID, without the risk of inducing significant 5-HT syndrome. Propranolol is thought to affect dyskinesia by decreasing the firing rate in ChIs, leading to a decrease in ACh levels and consequently to a decrease in DA release. Although in the clinical practice STN-DBS is the only stimulation applied in advanced stage of PD, literature suggests that DBS can be applied to two regions, STN and GPi. Stimulation of the GPi produces better results, although STN-DBS is also effective in controlling symptoms of both PD and LID. Stimulation of areas above the STN gives better results than stimulation of the STN alone.

Author Contributions: Conceptualization was performed by M.H. Methodology was performed by M.H., J.H., A.K.-M., A.G. Software by M.H., J.H., A.K.-M., A.G. Validation was performed by M.H., J.H., A.K.-M., A.G. Formal analysis was performed by A.K.-M., A.G. Investigation was performed by M.H., J.H., A.K.-M., A.G. Resources were provided by M.H., J.H., A.K.-M., A.G. Data curation was performed by M.H., J.H., A.K.-M., A.G. Writing-original draft preparation was performed by M.H., J.H., A.K.-M., A.G. Writing - review and editing was performed by M.H., J.H., A.K.-M., A.G. Supervision was performed by A.K.-M., A.G. Project administration was performed by A.G. Funding acquisition was performed by A.G. All authors have read and agreed to the published version of the manuscript.

Funding: This research received no external funding.

Conflicts of Interest: The authors declare no conflict of interest.

\section{References}

1. Albin, R.L.; Leventhal, D.K. The Missing, The Short, and The Long: L-Dopa Responses and Dopamine Actions. Ann Neurol 2018, 82(1), 4-19

2. Ockleford, C.; Adriaanse, P. Investigation into experimental toxicological properties of plant protection products having a potential link to Parkinson's disease and childhood leukaemia. EFSA J 2017, 15(3), e04691

3. Eusebi, P.; Romoli, M. Risk factors of levodopa-induced dyskinesia in Parkinson's disease: results from the PPMI cohort. NPJ Parkinsons Dis 2018, 4, 33

4. Calabresi, P.; Standaert, D.G. Dystonia and levodopa-induced dyskinesias in Parkinson's disease: Is there a connection? Neurobiol Dis 2019, 132, 104579

5. Fletcher, E.J.R.; Finlay, C.J. Neuroanatomical and Microglial Alterations in the Striatum of Levodopa-Treated, Dyskinetic HemiParkinsonian Rats. Front Neurosci 2020, 14, 567222

6. Ueno, T.; Nishijima H. Spine Enlargement of Pyramidal Tract-Type Neurons in the Motor Cortex of a Rat Model of LevodopaInduced Dyskinesia. Front Neurosci 2017, 11, 206 
7. Zhi, Y.; Wang, M. The increased gray matter volumes of precentral gyri in Parkinson's disease patients with diphasic dyskinesia. Aging (Albany NY) 2019, 11(21), 9661-9671

8. Lidenbach, D.; Conti, M.M. Alterations in primary motor cortex neurotransmission and gene expression in hemi-Parkinsonian rats with drug-induced dyskinesia. Neuroscience 2015, 310, 12-26

9. Chung, S.J.; Soo Yoo, H. White matter hyperintensities and risk of levodopa-induced dyskinesia in Parkinson's disease. Ann Clin Transl Neurol 2020, 7(2), 229-238

10. Li, X.; Zhuang, P. Altered Neuronal Firing Pattern of the Basal Ganglia Nucleus Plays a Role in Levodopa-Induced Dyskinesia in Patients with Parkinson's Disease. Front Hum Neurosci 2015; 9, 630

11. Porras, G.; De Deurwaerdere, P. L-dopa-induced dyskinesia: beyond an excessive dopamine tone in the striatum. Sci Rep 2014, 4,3730

12. Aristeta, A.; Azkona, G. The Role of the Subthalamic Nucleus in L-DOPA Induced Dyskinesia in 6-Hydroxydopamine Lesioned Rats. PloS One 2012, 7(8), e42652

13. Aristieta, A.; Ruiz-Ortega, J.A. Acute L-DOPA administration reverses changes in firing pattern and low frequency oscillatory activity in the entopeduncular nucleus from long term L-DOPA treated 6-OHDA-lesioned rats. Exp Neurol 2019, 322,113036

14. Soo Yoo, H.; Choi, Y.H. Cerebellar connectivity in Parkinson's disease with levodopa-induced dyskinesia. Ann Clin Transl Neurol 2019, 6(11), 2251-2260

15. Lewis, M.M.; Du, G. Higher iron in the red nucleus marks Parkinson's dyskinesia. Neurobiol Aging 2013, 34(5), 1497-1503

16. Jourdain, V.A.; Schindlbeck, K.A. Increased putamen hypercapnic vasoreactivity in levodopa-induced dyskinesia. JCI Insight 2017, 2(20), e96411

17. Jourdain, V.A.; Tang, Ch.C. Flow-metabolism dissociation in the pathogenesis of levodopa-induced dyskinesia. JCI Insight 2016, 1(15), e86615

18. Aljuaid, M., Booth, S. Blood Flow and Glucose Metabolism Dissociation in the Putamen Is Predictive of Levodopa Induced Dyskinesia in Parkinson's Disease Patients. Front Neurol 2019, 10, 1217

19. Andersen, A.D.; Blaabjerg, M. Cerebrospinal fluid levels of catecholamines and its metabolites in Parkinson's disease: effect of 1-DOPA treatment and changes in levodopa-induced dyskinesia. J Neurochem 2017, 141(4), 614-625

20. Yang, Ch.; Zhang, T. Brain-Region Specific Metabolic Abnormalities in Parkinson's Disease and Levodopa-Induced Dyskinesia. Front Aging Neurosci 2020, 12, 75

21. Ryu, H.S.; Park, K.W. Genomic Analysis Identifies New Loci Associated With Motor Complications in Parkinson's Disease. Front Neurol 2020, 11, 570

22. Ivanova, S.A.; Loonen, A.J.M. NMDA receptor genotypes associated with the vulnerability to develop dyskinesia. Transl Psychiatry 2012, 2(1), e67

23. Kakinuma, S.; Beppu, M. Monoamine oxidase B rs1799836 G allele polymorphism is a risk factor for early development of levodopa-induced dyskinesia in Parkinson's disease. eNeurologicalSci 2020, 19, 100239

24. Han, C.-L.; Liu, Y.-P. Integrated transcriptome expression profiling reveals a novel lncRNA associated with 1-DOPA-induced dyskinesia in a rat model of Parkinson's disease. Aging (Albany NY) 2020, 12(1), 718-739

25. Heiman, M.; Heilbut, A. Molecular adaptations of striatal spiny projection neurons during levodopa-induced dyskinesia. Proc Natl Acad Sci U S A 2014, 111(12), 4578-4583

26. Figge, D.A.; Eskow Jaunarajs, K.L. Dynamic DNA Methylation Regulates Levodopa-Induced Dyskinesia. J Neurosci 2016, 36(24), 6514-6524

27. Figge, D.A.; Standaert, D.G. Dysregulation of BET proteins in levodopa-induced dyskinesia. Neurobiol. Dis 2017, 102, 125-132

28. Ueno, S.; Oyama, G. A Retrospective Imaging Evaluation of Presynaptic Dopaminergic Degeneration in Multiple System Atrophy with Levodopa Induced Dyskinesia. Tremor Other Hyperkinet Mov (N Y) 2020, 10, 6

29. Jeong, E.H.; Sunwoo, M.K. Serial I-123-FP-CIT SPECT Image Findings of Parkinson's Disease Patients With Levodopa-Induced Dyskinesia. Front Neurol 2018, 9, 1133

30. Herz, D.M.; Haagensen, B.N. Abnormal dopaminergic modulation of striato-cortical networks underlies levodopa-induced dyskinesias in humans. Brain 2015, 138(6), 1658-1666

31. Herz, D.M.; Haagensen, B.N. The acute brain response to levodopa heralds dyskinesias in Parkinson disease. Ann Neurol 2014, 75(6), 829-836

32. Girasole, A.E.; Lum, M.Y. A Subpopulation of Striatal Neurons Mediates Levodopa-Induced Dyskinesia. Neuron 2018, 97(4), 787-795.e6

33. Ryan, M.B.; Bair-Marshall, Ch. Aberrant Striatal Activity in Parkinsonism and Levodopa-Induced Dyskinesia. Cell Rep 2018, 23(12), 3438-3446.e5

34. Parker, J.G.; Marshall, J.D. Diametric neural ensemble dynamics in parkinsonian and dyskinetic states. Nature 2019, 557(7704), 177-182

35. Li, L.; Zhou, F.M. Parallel dopamine D1 receptor activity dependence of 1 -Dopa-induced normal movement and dyskinesia in mice. Neuroscience 2013, 236, 66-76

36. Singh, A.; Liang, L. Dopamine regulates distinctively the activity patterns of striatal output neurons in advanced parkinsonian primates. J Neurophysiol 2015, 113(5), 1533-1544

37. Alberico, S.L.; Kim, Y.Ch. Axial levodopa-induced dyskinesias and neuronal activity in the dorsal striatum. Neuroscience 2017, $343,240-249$ 
38. Belić, J.J; Halje, P. Untangling Cortico-Striatal Connectivity and Cross-Frequency Coupling in L-DOPA-Induced Dyskinesia. Front Syst Neurosci 2016, 10, 26

39. Halje, P. Tamte, M. Levodopa-Induced Dyskinesia Is Strongly Associated with Resonant Cortical Oscillations. J Neurosci 2012, 32(47), 16541-16551

40. Thiele, S.L.; Chen, B. Selective loss of bi-directional synaptic plasticity in the direct and indirect striatal output pathways accompanies generation of parkinsonism and 1-DOPA induced dyskinesia in mouse models. Neurobiol Dis 2014, 71, 334-344

41. Perez, X.A.; Zhang, D. Striatal D1 medium spiny neuron activation induces dyskinesias in parkinsonian mice. Mov Disord 2017, $32(4), 538-548$

42. Shen, W.; Ren, W. Striatal Kir2 K+ channel inhibition mediates the antidyskinetic effects of amantadine. J Clin Invest 2020, 130(5), 2593-2601

43. Okada, M.; Nakao, R. Microdialysis with radiometric monitoring of $-\left[\beta-{ }^{11} \mathrm{C}\right] \mathrm{DOPA}$ to assess dopaminergic metabolism: effect of inhibitors of -amino acid decarboxylase, monoamine oxidase, and catechol-O-methyltransferase on rat striatal dialysate. $J$ Cereb Blood Flow Metab 2011, 31(1), 124-131

44. Loonen, A.J.M.; Ivanova, S.A. Polymorphisms of Catechol-O-Methyl Transferase (COMT) Gene in Vulnerability to LevodopaInduced Dyskinesia. J Pharm Pharm Sci 2018, 21(1), 340

45. Torkaman-Boutorabi, A.; Shahidi, G.A. The catechol-O-methyltransferase and monoamine oxidase B polymorphisms and levodopa therapy in the Iranian patients with sporadic Parkinson's disease. Acta Neurobiol Exp 2012, 72, 272-282

46. Santini, E.; Feyder, M. Dopamine- and cAMP-regulated Phosphoprotein of 32-kDa (DARPP-32)-dependent Activation of Extracellular Signal-regulated Kinase (ERK) and Mammalian Target of Rapamycin Complex 1 (mTORC1) Signaling in Experimental Parkinsonism. J Biol Chem 2012, 287(33), 27806-27812

47. Calabrese, V.; Di Maio, A. Rapamycin, by Inhibiting mTORC1 Signaling, Prevents the Loss of Striatal Bidirectional Synaptic Plasticity in a Rat Model of L-DOPA-Induced Dyskinesia. Front Aging Neurosci 2020, 12, 230

48. Wu, N.; Wan, Y. The abnormal activation of D1R/Shp-2 complex involved in levodopa-induced dyskinesia in 6-hydroxydopamine-lesioned Parkinson's rats. Neuropsychiatr Dis Treat 2018, 14 1779-1786

49. Jones-Tabah, J.; Mohammad, H. Dopamine D1 receptor signalling in dyskinetic Parkinsonian rats revealed by fiber photometry using FRET-based biosensors. Sci Rep 2020, 10(1), 14426

50. Fieblinger, J.; Sebastianutto, I. Mechanisms of Dopamine D1 Receptor-Mediated ERK1/2 Activation in the Parkinsonian Striatum and Their Modulation by Metabotropic Glutamate Receptor Type 5. J Neurosci 2014, 34(13), 4728-4740

51. Alcacer, C.; Santini, E. Goolf Mutation Allows Parsing the Role of cAMP-Dependent and Extracellular Signal-Regulated KinaseDependent Signaling in 1-3,4-Dihydroxyphenylalanine-Induced Dyskinesia. J Neurosci 2012, 32(17), 5900-5910

52. Goto, S. Striatal Goolf/cAMP Signal-Dependent Mechanism to Generate Levodopa-Induced Dyskinesia in Parkinson's Disease. Front Cell Neurosci 2017, 11, 364

53. Södersten, E.; Feyder, M. Dopamine Signaling Leads to Loss of Polycomb Repression and Aberrant Gene Activation in Experimental Parkinsonism. PLoS Genet 2014, 10(9), e1004574

54. Sellnow, R.C.; Steece-Collier, K. Striatal Nurr1 Facilitates the Dyskinetic State and Exacerbates Levodopa-Induced Dyskinesia in a Rat Model of Parkinson's Disease. J Neurosci 2020, 40(18), 3675-3691

55. Stanic, J.; Mellone, M. LRRK2 phosphorylation level correlates with abnormal motor behaviour in an experimental model of levodopa-induced dyskinesias. Mol Brain.2016, 9, 53

56. Divitio, C.B.; Steece-Collier, K. Loss of VGLUT3 Produces Circadian-Dependent Hyperdopaminergia and Ameliorates Motor Dysfunction and 1-Dopa-Mediated Dyskinesias in a Model of Parkinson's Disease. J Neurosci 2015, 35(45), 14983-14999

57. Porras, G.; Berthet, A. PSD-95 expression controls 1-DOPA dyskinesia through dopamine D1 receptor trafficking. J Clin Invest 2012, 122(11), 3977-3989

58. Berthet, A.; Bezard, E. 1-DOPA Impairs Proteasome Activity in Parkinsonism through D1 Dopamine Receptor. J Neurosci 2012, 32(2), 681-691

59. Zhang, S.F.; Xie, C.L. Interactions of CaMKII with dopamine D2 receptors: roles in levodopa-induced dyskinesia in 6-hydroxydopamine lesioned Parkinson's rats. Sci Rep 2014, 4, 6811

60. Albarrán-Bravo, S.; Ávalos-Fuentes, J.A. Severity of Dyskinesia and D3R Signaling Changes Induced by L-DOPA Treatment of Hemiparkinsonian Rats Are Features Inherent to the Treated Subjects. Biomolecules 2019, 9(9), 431

61. Solís, O.; Garcia-Montes, J.R. Dopamine D3 Receptor Modulates 1-DOPA-Induced Dyskinesia by Targeting D1 Receptor-Mediated Striatal Signaling. Cereb Cortex 2017, 27(1), 435-446

62. Payer, D.E.; Guttman, M. D3 dopamine receptor-preferring $\left[{ }^{11} \mathrm{C}\right]$ PHNO PET imaging in Parkinson patients with dyskinesia. Neurology 2016, 86(3), 224-230

63. Castello, J.; Cortés, M. The Dopamine D5 receptor contributes to activation of cholinergic interneurons during L-DOPA induced dyskinesia. Sci Rep 2020, 10, 2542

64. Bido, S.; Solari, N. Differential involvement of Ras-GRF1 and Ras-GRF2 in L-DOPA-induced dyskinesia. Ann Clin Transl Neurol 2015, 2(6), 662-678

65. Fasano, S.; Bezard, E. Inhibition of Ras-guanine nucleotide-releasing factor 1 (Ras-GRF1) signaling in the striatum reverts motor symptoms associated with 1-dopa-induced dyskinesia. Proc Natl Acad Sci U S A 2010, 107(50), 21824-21829

66. Eshraghi, M.; Ramírez-Jarquín, U.N. RasGRP1 is a causal factor in the development of l-DOPA-induced dyskinesia in Parkinson's disease. Neuroscience 2020, 6(18), eeaz7001 
67. Cerovic, M.; Bagetta, V. Derangement of Ras-guanine nucleotide-releasing factor 1 (Ras-GRF1) and extracellular signal-regulated kinase (ERK) dependent striatal plasticity in L-DOPA-induced dyskinesia. Biol Psychiatry 2015, 77(2) 106-115

68. Ruiz-DeDiego, I.; Fasano, S. Genetic enhancement of Ras-ERK pathway does not aggravate L-DOPA-induced dyskinesia in mice but prevents the decrease induced by lovastatin. Sci Rep 2018, 8, 15381

69. Beck, G.; Singh, A. Role of striatal $\triangle$ FosB in l-Dopa-induced dyskinesias of parkinsonian nonhuman primates. Proc Natl Acad Sci U S A 2019, 116(37), 18664-18672

70. Cortés, M.; Malave, L. CK2 Oppositely Modulates 1-DOPA-Induced Dyskinesia via Striatal Projection Neurons Expressing D1 or D2 Receptors. J Neurosci 2017, 37(49), 11930-11946

71. Lindenbach, D.; Conti, M.M. Alterations in primary motor cortex neurotransmission and gene expression in hemi-parkinsonian rats with drug-induced dyskinesia. Neuroscience 2015, 310, 12-26

72. Bastide, M.F.; Glangetas, C. Involvement of the bed nucleus of the stria terminalis in L-Dopa induced dyskinesia. Sci Rep 2017, 7, 2348

73. Padovan-Neto, F.E.; Ferreira, N.R. Anti-dyskinetic effect of the neuronal nitric oxide synthase inhibitor is linked to decrease of FosB/DeltaFosB expression. Neurosci Lett 2013, 541, 126-131

74. Stanic, J.; Mellone, M. Rabphilin 3A: A novel target for the treatment of levodopa-induced dyskinesias. Neurobiol. Dis 2017, 108, 54-64

75. Ba, M.; Kong, M. Postsynaptic density protein 95-regulated NR2B tyrosine phosphorylation and interactions of Fyn with NR2B in levodopa-induced dyskinesia rat models. Drug Des Devel Ther 2015, 9, 199-206

76. Lindenbach, D.; Conti, M.M. The Role of Primary Motor Cortex (M1) Glutamate and GABA Signaling in 1-DOPA-Induced Dyskinesia in Parkinsonian Rats. J Neurosci 2016, 36(38), 9873-9887

77. Brumberg, J.; Küsters, S. Cholinergic activity and levodopa-induced dyskinesia: a multitracer molecular imaging study. Ann Clin Transl Neurol 2017, 4(9), 632-639.

78. Brugnoli, A.; Pisanò, C.A. Striatal and nigral muscarinic type 1 and type 4 receptors modulate levodopa-induced dyskinesia and striato-nigral pathway activation in 6-hydroxydopamine hemilesioned rats. Neurobiol. Dis 2020, 144, 105044

79. Shen, W.; Plotkin, J.L. M4 Muscarinic Receptor Signaling Ameliorates Striatal Plasticity Deficits in Models of L-DOPA-Induced Dyskinesia. Neuron 2015, 88(4), 762-763

80. Sahin, G.; Thompson, L.H. Differential Dopamine Receptor Occupancy Underlies L-DOPA-Induced Dyskinesia in a Rat Model of Parkinson's Disease. PLoS One 2014, 9(3), e90759

81. Bishop, C.; George, J.A. Serotonin transporter inhibition attenuates 1-DOPA-induced dyskinesia without compromising 1-DOPA efficacy in hemi-parkinsonian rats. Eur J Neurosci 2012, 36(6), 2839-2848

82. Miguelez, C.; Navailles, S. The acute and long-term L-DOPA effects are independent from changes in the activity of dorsal raphe serotonergic neurons in 6-OHDA lesioned rats. Br J Pharmacol 2016, 173(13), 2135-2146

83. Santos-Lobato, B.L.; Schumacher-Schuh, A.F. Diagnostic prediction model for levodopa-induced dyskinesia in Parkinson's disease. Arq Neuropsiquiatr. 2020, 78(4), 206-216

84. Hong, J.Y.; Sunwoo, M.K. Rapid drug increase and early onset of levodopa-induced dyskinesia in Parkinson's disease. PLoS One 2020, 15(8), e0237472

85. Turcano, P.; Mielke, M.M. Levodopa-induced dyskinesia in Parkinson disease; A population-based cohort study. Neurology 2018, 91(24), e2238-e2243

86. Lin, M.M.; Laureno, R. Less Pulsatile Levodopa Therapy (6 Doses Daily) Is Associated with a Reduced Incidence of Dyskinesia. J Mov Disord 2019, 12(1), 37-42

87. Poewe, W.; Chaudhuri, K.R. Levodopa-carbidopa intestinal gel in a subgroup of patients with dyskinesia at baseline from the GLORIA Registry. Neurodegener Dis Manag 2019, 9(1), 39-46

88. Ramlackhansingh, A.F.; Bose, S.K. Adenosine 2A receptor availability in dyskinetic and nondyskinetic patients with Parkinson disease. Neurology 2011, 76(21), 1811-1816

89. Wills, A.-M.A. Eberly, S. Caffeine consumption and risk of dyskinesia in CALM-PD. Mov Disord 2013, 28(3), 380-383

90. Riggare, S.; Unruh, K.T. Patient-driven N-of-1 in Parkinson's Disease; Lessons Learned from a Placebo-controlled Study of the Effect of Nicotine on Dyskinesia. Methods Inf Med 2017, 56(Suppl 1), e123-e128

91. Wan, Y.; Wu, N. Levodopa/Benserazide Loaded Microspheres Alleviate L-dopa Induced Dyskinesia through Preventing the Over-Expression of D1R/Shp-2/ERK1/2 Signaling Pathway in a Rat Model of Parkinson's Disease. Front Aging Neurosci 2017, 9, 331

92. Yang, X.; Zheng, R. Controlled-release levodopa methyl ester/benserazide-loaded nanoparticles ameliorate levodopa-induced dyskinesia in rats. Int J Nanomedicine 2012, 7, 2077-2086

93. Xie, C.I.; Wang, W.W. Levodopa/benserazide microsphere (LBM) prevents L-dopa induced dyskinesia by inactivation of the DR1/PKA/P-tau pathway in 6-OHDA-lesioned Parkinson's rats. Sci Rep 2014, 4, 7506

94. Yang, X.; Chen, Y. Levodopa/benserazide microspheres reduced levodopa-induced dyskinesia by downregulating phosphorylated GluR1 expression in 6-OHDA-lesioned rats. Drug Des Devel Ther 2012, 6, 341-347

95. Cao, X.; Hou, D. Effects and molecular mechanism of chitosan-coated levodopa nanoliposomes on behavior of dyskinesia rats. Biol Res 2016, 49, 32

96. LeWitt, P.A.; Verhagen, Metman L. Effect of Concomitant Medications on the Safety and Efficacy of Extended-Release Carbidopa-Levodopa (IPX066) in Patients With Advanced Parkinson Disease: A Post Hoc Analysis. Clin Neuropharmacol 2018, 41(2), $47-55$ 
97. Stocchi, F.; Hsu, A. Comparison of IPX066 with carbidopa-levodopa plus entacapone in advanced PD patients. Parkinsonism Relat Disord 2014, 20(12), 1335-1340

98. Bido, S.; Marti, M. Amantadine attenuates levodopa-induced dyskinesia in mice and rats preventing the accompanying rise in nigral GABA levels. J. Neurochem 2011, 118, 1043-1055

99. Pahwa, R.; Tanner, C.M. Amantadine extended release for levodopa-induced dyskinesia in Parkinson's disease (EASED Study). Mov Disord 2015, 30(6), 788-795

100. Oertel, W.; Eggert, K. Randomized, placebo-controlled trial of ADS-5102 (amantadine) extended-release capsules for levodopainduced dyskinesia in Parkinson's disease (EASE LID 3). Mov Disord 2017, 32(12), 1701-1709

101. Tanner, C.M.; Pahwa, R. EASE LID 2: A 2-Year Open-Label Trial of Gocovri (Amantadine) Extended Release for Dyskinesia in Parkinson's Disease. J Parkinsons Dis 2020, 10(2), 543-558

102. Hauser, R.A.; Pahwa, R. ADS-5102 (Amantadine) Extended-Release Capsules for Levodopa-Induced Dyskinesia in Parkinson's Disease (EASE LID 2 Study): Interim Results of an Open-Label Safety Study. J Parkinsons Dis 2017, 7(3), 511-522

103. Pahwa, R.; Tanner, C.M. ADS-5102 (Amantadine) Extended-Release Capsules for Levodopa-Induced Dyskinesia in Parkinson Disease (EASE LID Study). JAMA Neurol 2017, 74(8), 941-949

104. Solis, O.; Garcia-Montes, J.R. Dopamine D3 Receptor Modulates 1-DOPA-Induced Dyskinesia by Targeting D1 Receptor-Mediated Striatal Signaling. Cereb Cortex 2017, 27(1), 435-446

105. Xu, W.; Wang, X. Functional Characterization of a Novel Series of Biased Signaling Dopamine D3 Receptor Agonists. ACS Chem. Neurosci 2017, 8(3), 486-500

106. Utsumi, H.; Okuma, Y. Evaluation of the Efficacy of Pramipexole for Treating Levodopa-induced Dyskinesia in Patients with Parkinson's Disease. Intern Med 2013, 52, 325-332

107. Visanji, N.P.; Fox, S.H. Dopamine D3 receptor stimulation underlies the development of L-DOPA-induced dyskinesia in animal models of Parkinson's disease. Neurobiol Dis 2009, 35(2), 184-192

108. Svenningsson, P.; Johansson, A. Safety and tolerability of IRL790 in Parkinson's disease with levodopa-induced dyskinesia-a phase $1 \mathrm{~b}$ trial. NPJ Parkinsons Dis 2018, 4, 35

109. Borgohain, R.; Szasz, J. Randomized trial of safinamide add-on to levodopa in Parkinson's disease with motor fluctuations. Mov Disord 2014, 29(2), 229-237

110. Borgohain, R.; Szasz, J. Two-year, randomized, controlled study of safinamide as add-on to levodopa in mid to late Parkinson's disease. Mov Disord 2014, 29(10), 1273-80

111. Cattaneo, C.; Jost, W.H. Long-Term Efficacy of Safinamide on Symptoms Severity and Quality of Life in Fluctuating Parkinson's Disease Patients. J Parkinsons Dis 2020, 10(1), 89-97

112. Gardoni, F.; Morari, M. Safinamide Modulates Striatal Glutamatergic Signaling in a Rat Model of Levodopa-Induced Dyskinesia. J Pharmacol Exp Ther 2018, 367(3), 442-451

113. Hernandez, M.; Walsch, M. Serotonin Syndrome in the Emergency Department. Cureus 2019, 11(12), e6307

114. Sagarduy, A.; Llorente, J. Buspirone requires the intact nigrostriatal pathway to reduce the activity of the subthalamic nucleus via 5-HT1A receptors. Exp Neurol 2016, 277, 35-45

115. Vegas-Suárez, S.; Pisanò, C.A. 6-Hydroxydopamine lesion and levodopa treatment modify the effect of buspirone in the substantia nigra pars reticulata. Br J Pharmacol 2020, 177(17), 3957-3974

116. Nahimi, A.; Høltzermann, M. Serotonergic modulation of receptor occupancy in rats treated with L-DOPA after unilateral 6OHDA lesioning. J Neurochem 2012, 120(5), 806-17

117. Dupre, K.B.; Cruz, A.V. Effects of L-dopa priming on cortical high beta and high gamma oscillatory activity in a rodent model of Parkinson's disease. Neurobiol Dis 2016, 86, 1-15

118. Lindenbach, D.; Palumbo, N. Side effect profile of 5-HT treatments for Parkinson's disease and L-DOPA-induced dyskinesia in rats. Br J Pharmacol 2014, 172(1), 119-30

119. Bhide, N.; Lindenbach, B. The effects of BMY-14802 against L-DOPA- and dopamine agonist-induced dyskinesia in the hemiparkinsonian rat. Psychopharmacology (Berl) 2013, 227(3), 533-44

120. Svenningsson, P.; Rosenblad, C. Eltoprazine counteracts 1-DOPA-induced dyskinesias in Parkinson's disease: a dose-finding study. Brain 2015, 138(4), 963-973

121. Aboulghasemi, N.; Jahromy, M.H. Anti-dyskinetic efficacy of 5-HT3 receptor antagonist in the hemi-parkinsonian rat model. IBRO Rep 2019, 6, 40-44

122. Lindenbach, D.; Ostock, C.Y. Behavioral and Cellular Modulation of 1-DOPA-Induced Dyskinesia by $\beta$-Adrenoceptor Blockade in the 6-Hydroxydopamine-Lesioned Rat. J Pharmacol Exp Ther 2011, 337(3), 755-765

123. Bhide, N.; Lindenbach, D. Effects of the beta-adrenergic receptor antagonist Propranolol on dyskinesia and L-DOPA-induced striatal DA efflux in the hemi-parkinsonian rat. J Neurochem 2015, 134(2), 222-32

124. Shi, Z.; Barnford, I.J. Propranolol Relieves L-Dopa-Induced Dyskinesia in Parkinsonian Mice. Brain Sci 2020, 10(12), 903

125. Seppä, T.; Ruotsalainen, M. Effect of acute nicotine administration on striatal dopamine output and metabolism in rats kept at different ambient temperatures. Br J Pharmacol 2000, 130(5), 1147-1155

126. Bodia, T.; McIntosh, J.M. The nicotine-mediated decline in 1-dopa-induced dyskinesias is associated with a decrease in striatal dopamine release. J Neurochem 2013, 125(2), 291-302

127. Leino, S.; Kohtala, S. Dyskinesia and brain-derived neurotrophic factor levels after long-term levodopa and nicotinic receptor agonist treatments in female mice with near-total unilateral dopaminergic denervation. BMC Neurosci 2018, 19, 77 
128. Teng, L.; Hong, F. Compound Formula Rehmannia alleviates levodopa-induced dyskinesia in Parkinson's disease. Neural Regen Res 2014, 9(4), 407-412

129. Ogawa, M.; Zhou, Y. Intrastriatal Memantine Infusion Dampens Levodopa-Induced Dyskinesia and Motor Deficits in a Mouse Model of Hemiparkinsonism. Front Neurol 2019, 10, 1258

130. Cui, G.; Yang, X. Ranitidine reduced levodopa-induced dyskinesia in a rat model of Parkinson's disease. Neuropsychiatr Dis Treat 2014, 10, 39-46

131. Shi, H.; Yang, X. Ranitidine reduced levodopa-induced dyskinesia by remodeling neurochemical changes in hemiparkinsonian model of rats. Neuropsychiatr Dis Treat 2015, 11, 1331-1337

132. Mestre, T.A.; Shah, B.B. Famotidine, a Histamine H2 Receptor Antagonist, Does Not Reduce Levodopa-Induced Dyskinesia in Parkinson's Disease: A Proof-of-Concept Study. Mov Disord Clin Pract 2014, 1(3), 219-224

133. Johansson, P.A.; Andersson, M. Alterations in cortical and basal ganglia levels of opioid receptor binding in a rat model of $1-$ DOPA-induced dyskinesia. Neurobiol Dis 2001, 8(2), 220-239

134. Pots, L.F.; Park, E.S. Dual $\kappa$-agonist/ $\mu$-antagonist opioid receptor modulation reduces levodopa-induced dyskinesia and corrects dysregulated striatal changes in the nonhuman primate model of Parkinson disease. Ann Neurol 2015, 77(6), 930-41

135. Bartlett, M.J.; So, L.Y. Highly-selective $\mu$-opioid receptor antagonism does not block L-DOPA-induced dyskinesia in a rodent model. BMC Res Notes 2020, 13, 149

136. Bezard, E.; Li, Q. $\mu$ Opioid Receptor Agonism for L-DOPA-Induced Dyskinesia in Parkinson's Disease. J Neurosci 2020, 40(35), 6812-6819

137. Arcuri, L.; Novello, S. Anti-Parkinsonian and anti-dyskinetic profiles of two novel potent and selective nociceptin/orphanin FQ receptor agonists. Br J Pharmacol 2018, 175(5), 782-796

138. Niccolini, F.; Foltynie, T. Loss of phosphodiesterase 10A expression is associated with progression and severity in Parkinson's disease. Brain 2015, 138(10), 3003-3015

139. Beck, G.; Maehara, S. A Selective Phosphodiesterase 10A Inhibitor Reduces L-Dopa-Induced Dyskinesias in Parkinsonian Monkeys. Mov Disord 2018, 33(5), 805-814

140. Sellnow, R.C.; Newman, J.H. Regulation of dopamine neurotransmission from serotonergic neurons by ectopic expression of the dopamine D2 autoreceptor blocks levodopa-induced dyskinesia. Acta Neuropathol Commun 2019, 7, 8

141. Marongiu, R. Arango-Lievano, M. Gene therapy blockade of dorsal striatal p11 improves motor function and dyskinesia in parkinsonian mice. Proc Natl Acad Sci U S A 2016, 113(5), 1423-1428

142. Breger, L.S.; Kienle, K. Influence of chronic L-DOPA treatment on immune response following allogeneic and xenogeneic graft in a rat model of Parkinson's disease. Brain Behav Immun 2017, 61, 155-164

143. Ahmed, M.R.; Bychkov, E. Overexpression of GRK6 rescues 1-DOPA-induced signaling abnormalities in the dopamine-depleted striatum of hemiparkinsonian rats. Exp Neurol 2015, 266, 42-54

144. Zhang, X.R.; Zhang, Z.R. $\beta$-arrestin2 alleviates L-dopa-induced dyskinesia via lower D1R activity in Parkinson's rats. Aging (Albany NY) 2019, 11(24), 12315-12327

145. Urs, N.M.; Bido, S. Targeting $\beta$-arrestin2 in the treatment of 1-DOPA-induced dyskinesia in Parkinson's disease. Proc Natl Acad Sci U S A 2015, 112(19), E2517-E2526

146. Park, H.-Y.; Kang, Y.-M. Inhibition of Adenylyl Cyclase Type 5 Prevents 1-DOPA-Induced Dyskinesia in an Animal Model of Parkinson's Disease. J Neurosci 2014, 34(35), 11744-11753

147. Leino S.; Koski S.K. Attenuated dopaminergic neurodegeneration and motor dysfunction in hemiparkinsonian mice lacking the $\alpha 5$ nicotinic acetylcholine receptor subunit. Neuropharmacology 2018, 138, 371-380

148. Yang, X.; Wu, N. Intrastriatal injections of KN-93 ameliorates levodopa-induced dyskinesia in a rat model of Parkinson's disease. Neuropsychiatr Dis Treat. 2013, 9, 1213-1220

149. Steece-Collier, K.; Stancat, J.A. Genetic silencing of striatal CaV1.3 prevents and ameliorates levodopa dyskinesia. Mov Disord 2019, 34(5), 697-707

150. Martinez, A.A.; Morgese, M.G. Activation of PPAR gamma receptors reduces levodopa-induced dyskinesias in 6-OHDA-lesioned rats. Neurobiol Dis 2015, 74, 295-304

151. Fan, S.-Y.; Wang, K.-L. Pallidal versus subthalamic nucleus deep brain stimulation for levodopa-induced dyskinesia. Ann Clin Transl Neurol 2020, 7(1), 59-68

152. Sobstyl, M.; Zabek, M. Unilateral Subthalamic Nucleus Stimulation in the Treatment of Asymmetric Parkinson's Disease with Early Motor Complications. Turk Neurosurg 2017, 27(2), 294-300

153. Kim, J.H.; Chang, W.S. Effect of Subthalamic Deep Brain Stimulation on Levodopa-Induced Dyskinesia in Parkinson's Disease. Yonsei Med. J 2015, 56(5), 1316-1321

154. Deuschl, G.; Schade-Brittinger, C. Neurostimulation for Parkinson's disease with early motor complications. N Engl J Med 2013, 368(21), 2038

155. Lohse, A.; Meder, D. Low-frequency transcranial stimulation of pre-supplementary motor area alleviates levodopa-induced dyskinesia in Parkinson's disease: a randomized cross-over trial. Brain Commun 2020, 2(2), fcaa147

156. Ni, Z.; Chen, R. Transcranial magnetic stimulation to understand pathophysiology and as potential treatment for neurodegenerative diseases. Transl Neurodegener. 2015, 4, 22 